\title{
Vantaxes do uso de Twitter na aula de italiano como LE
}

\author{
Tucci, Emiliana \\ Universidade da Coruña, Facultade de Filoloxía, \\ https://orcid.org/0000-0002-0127-6215
}

\section{RESUMO}

0 "Grupo de Innovación Educativa no Ensino-Aprendizaxe das Linguas e Literaturas Románicas" da Facultade de Filoloxía ten, entre outros obxectivos, fomentar o uso das redes sociais no ensino das linguas románicas. Neste artigo propoñemos os resultados da experiencia do uso de Twitter na aula da materia "Idioma Moderno 3: Italiano", na cal participaron todo o alumnado matriculado.

A implementación desta, e de máis redes sociais que adoptamos noutras disciplinas en anos anteriores, ten como obxectivo principal 0 achegamento á lingua italiana mediante unha ferramenta próxima e de uso común entre 0 estudantado. Esta aproximación puido ser posíbel grazas a unha planificación de actividades semanais cuxas finalidades atinxen a aspectos gramaticais como 0 uso do subxuntivo, 0 incremento do léxico, actividades de creación literaria e un achegamento ás linguas rexionais de Italia.

PALABRAS CLAVE: italiano LE; Twitter; ensinanza de linguas estranxeiras.

\section{CITA RECOMENDADA:}

Tucci, Emiliana (2020): Vantaxes do uso de Twitter na aula de italiano como LE. En De la Torre Fernández, E. (ed.) (2020). Contextos universitarios transformadores: Boas prácticas no marco dos GID. IV Xornadas de Innovación Docente. Cufie. Universidade da Coruña. A Coruña (págs. 251-262). DOI capítulo: https://doi.org/10.17979/spudc. 9788497497756.251 DOl libro: https://doi.org/10.17979/spudc.9788497497756 


\section{ABSTRACT}

The "Grupo de Innovación Educativa no Ensino-Aprendizaxe das Linguas e Literaturas Románicas" at the Faculty of Philology has, among other objectives, to increase the use of social networks in the teaching of Romance languages. In this article, we show the results of the experience of the use of Twitter in the course "Idioma Moderno 3: Italiano" in which all students have participated. The implementation of this and other social networks used in previous courses has as its main objective the approach to the Italian language through a closer and common tool among students. This closeness was possible thanks to a weekly programme of activities concerning grammatical aspects such as the use of the subjunctive, lexical increase, activities based on literary creation and an introduction to the regional languages of Italy.

KEY WORDS: Italian as a foreign language; Twitter; teaching foreign languages. 


\section{INTRODUCCIÓN}

Un dos obxectivos que nos propomos no "Grupo de Innovación Educativa no EnsinoAprendizaxe das Linguas e Literaturas Románicas" é o implemento das redes sociais na aula de Italiano. Xa nos anos anteriores puidemos manifestar os beneficios que Facebook e YouTube achegaron á hora de fortalecer algunhas habilidades lingüísticas, comunicativas e léxicas.

No ano 2018/2019, para a materia "Idioma Moderno 3: Italiano" suxerimos 0 uso dunha das redes sociais más en auxe entre os noso estudantado: Twitter. De xeito específico, creamos unha conta chamada "IdiomaModerno3_Italiano-18/19 (@Idioma3Italiano)" na cal participaron de xeito voluntario todos os compoñentes da materia.

A programación das actividades que ilustraremos a seguir foi desenvolvida dun xeito ad hoc para mellorar algunhas das habilidades exixidas no "Marco Común Europeo de Referencia para las linguas" (MCER) para a lingua italiana: i) mellorar a expresión escrita, ii) impulsar a capacidade de síntese na expresión escrita, iii) engadir coñecementos fraseolóxicos e iv) acrecentar os coñecementos do léxico.

Para acadar estes obxectivos propuxemos as seguintes actividades: 1) uso dos memes como instrumento didáctico; 2) comentario de aforismos; 3) comentario dun vídeo sobre 0 uso dos subxuntivos dos políticos italianos; 4) comentario de anuncios; 5) selección de música italiana; 6) actividades relacionadas coa cociña italiana; 7) aproximación ás linguas rexionais presentes no territorio italiano; 8) exercicios de creación literaria. No artigo, por razóns de espazo e de prioridade, imos presentar só algunhas delas.

\section{DESCRICIÓN DA EXPERIENCIA}

0 uso das redes sociais, neste caso específico de Twitter, no ámbito da educación considérase, xeralmente, como unha ferramenta útil para fortalecer coñecementos previamente desenvolvidos na aula. No caso dos idiomas, este instrumento adquire unha 
relevancia aínda máis considerábel se temos en conta as habilidades que o MCER require para a aprendizaxe das linguas. Saber expresarse por escrito, dominar o léxico e á vez facer que os temas de cultura sexan atractivos para o estudantado pode ser un reto para o profesorado. Por esas razóns, pensamos integrar o Twitter "na aula da materia "Idioma Moderno 3: Italiano", correspondente a un nivel B1.

No noso caso, os obxectivos principais que quixemos acadar son os seguintes:

- Fomentar o uso de Twitter en clase;

- Reforzar a idea de "informalidade" das actividades;

- Facer fincapé no uso didáctico da dita rede social.

- Fortalecer os coñecemento gramaticais explicados na clase;

- Introducir temas de cultura a través da reflexión gramatical;

- Achegar o alumnado á situación lingüística en Italia.

Para que estes obxectivos puideren ser alcanzados, propuxemos as actividades que describimos embaixo.

Actividade 1: televisión e fraseoloxía

Nesta primeira actividade, a docente publicou un meme dun cómico italiano (Imaxe 1) que muda un famoso refrán da lingua italiana: "Scherza con i fanti e lascia stare i fanti", que significa non mesturar o sagrado co profano, con "Scherza con i fanti e lascia stare gli elefanti". Os estudantes tiveron de encontrar a forma correcta do proverbio e explicar como chegaron á versión orixinal. 
Ciao ragazzi! Questa settimana dovrete commentare e correggere la frase di questo comico italiano, Nino Frassica. Qual è la forma corretta della frase? E come ci siete arrivati?? Buon lavoro!

Che Tempo Che Fa ob enetempocheta : 26 teb

II direllure di Nuvella Bella @inufrassicaufr a HCheTempuChera.

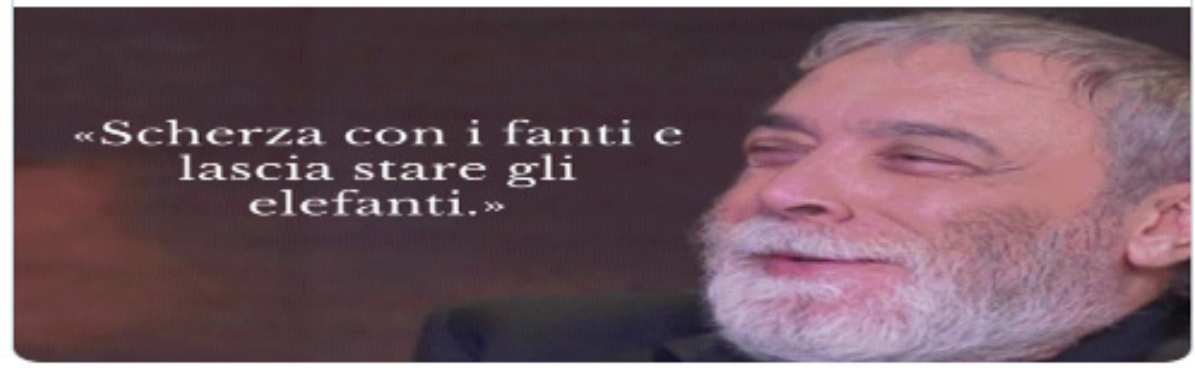

\section{In. 10 mar}

In rispusta a midioma 3 lisalianc

La torma corretta della trase e "Scherza con i tanti e lascia stare i santi", Pense que é un' alternetive divertente e anche intellicente! $\wedge$ me non mi sarebbe venuta in mente questa idea...

ऽ $\tau$

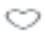

$\uparrow$

In risposta a coldiomasltaliano

la massima é "scher ca cen i farti, ma lascia stare i santi" ma pernso che e una alternativa alla originale pio divertente 3

Q Tl $⿻ 11 \%$

Ssposta a Qidiomasitaliano

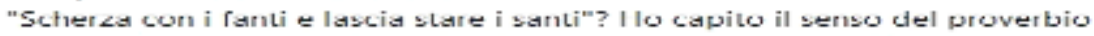
originale ma sono curiosa di sapere cosa abbia voluto dire frassica...
Q 1
$\tau>$
1
$\uparrow$

IdlomaModerno3:Itallano-18/19 coldiomäitalinno - 6 mar

Frassica e un mitoll! Vedi qualche video su YouTube se ti va!
cos
七7
() 1
$T$
It

Imaxe 2 
Actividade 2: a linguaxe dos anuncios

Nesta tarefa, os estudantes terán de ver, entender e comentar un dos anuncios máis icónicos dos anos 80 da televisión italiana, para alén de escoller un anuncio propio da súa nacionalidade que lles evoque recordos. Así mesmo, poderán entrar en contacto coa linguaxe específica da publicidade.

\section{IdiomaModernoz:Itallano-1 $8 / 19$}

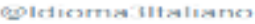

Ciao ragazzi! Questa settimana parliamo di pubblicità. Guardate questo video. Cosa ne pensate? $C^{\prime e}$ nel vostro paese qualche pubblicità che è passata alla storia come quella del pennello Cinghiale?

Buon lavoro!!!

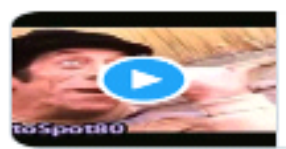

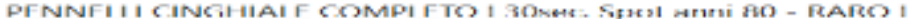

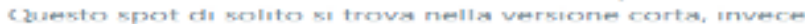

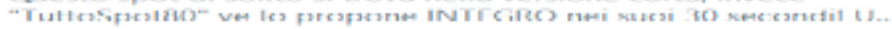
es? youtube,com

1:14 PM - 25 mar 2019. Twitter for Androld

ll Visualizza interazioni Tweet

\section{Imaxe 3}

\section{. 28 mar \\ In risposta a @Idioma3Italiano \\ Per me questa e stata la migliore pubblicita quando ero piccola. Ê la pubblicità di Cola-Cao più famosa in Spagna e sono sicura che la maggior parte dei ragazzi della mia etá lo ricorda ancora.}

\begin{tabular}{|c|c|}
\hline$\equiv$ & $\begin{array}{l}\text { Colacao (Surf) TV Spot } \\
\& \text { youtube.com }\end{array}$ \\
\hline & โ】 \\
\hline
\end{tabular}

Imaxe 4 


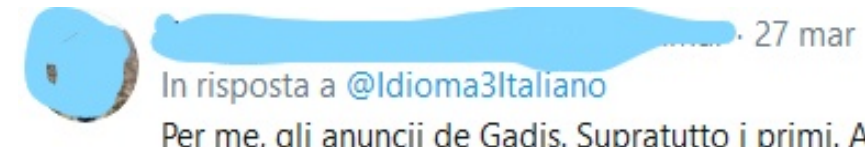

Per me, gli anuncii de Gadis. Supratutto i primi. Avevano una cosa che ti faceva alzarti piangendo e cantando Queixumes dos Pinos.

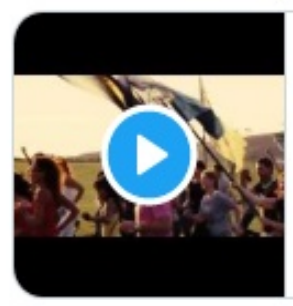

1000 anos máis

"Vivamos como galegos!" cumpre 5 anos e quixo

celebralo invitando a todos os galegos a participar n...

\&youtube.com

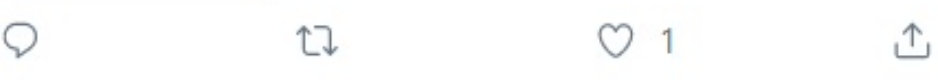

Imaxe 5

Actividade 3: a música, a memoria e a lingua

A música, como demostran varios estudosiii, é un potente medio para aprendizaxe dunha lingua. Ademais, 0 aspecto emocional da música, representa un estímulo máis para os estudantes se aproximaren á linguaxe musical. De feito, a finalidade deste exercicio é que publiquen un tweet coa súa canción italiana favorita, coa explicación da súa escolla.

\section{IdiomaModerno3:Italiano-18/19}

@Idioma3ltaliano

\section{Ciao ragazzi! Questa settimana parliamo di musica! Pubblicate nei commenti la vostra canzone italiana preferita e spiegate il motivo della vostra scelta. Buon lavoro!!!}
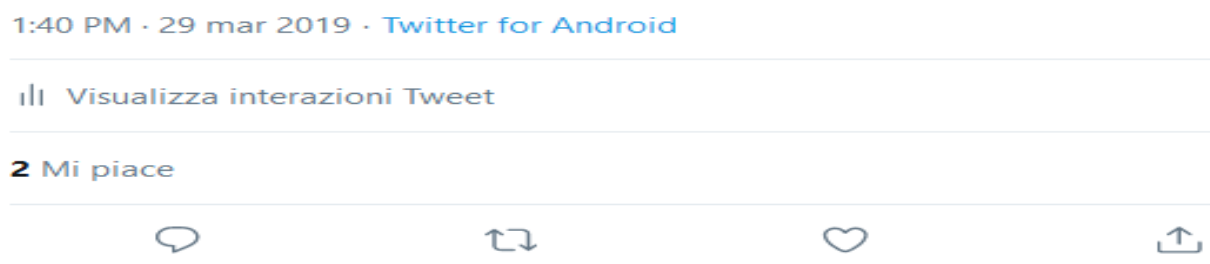

Imaxe 6 

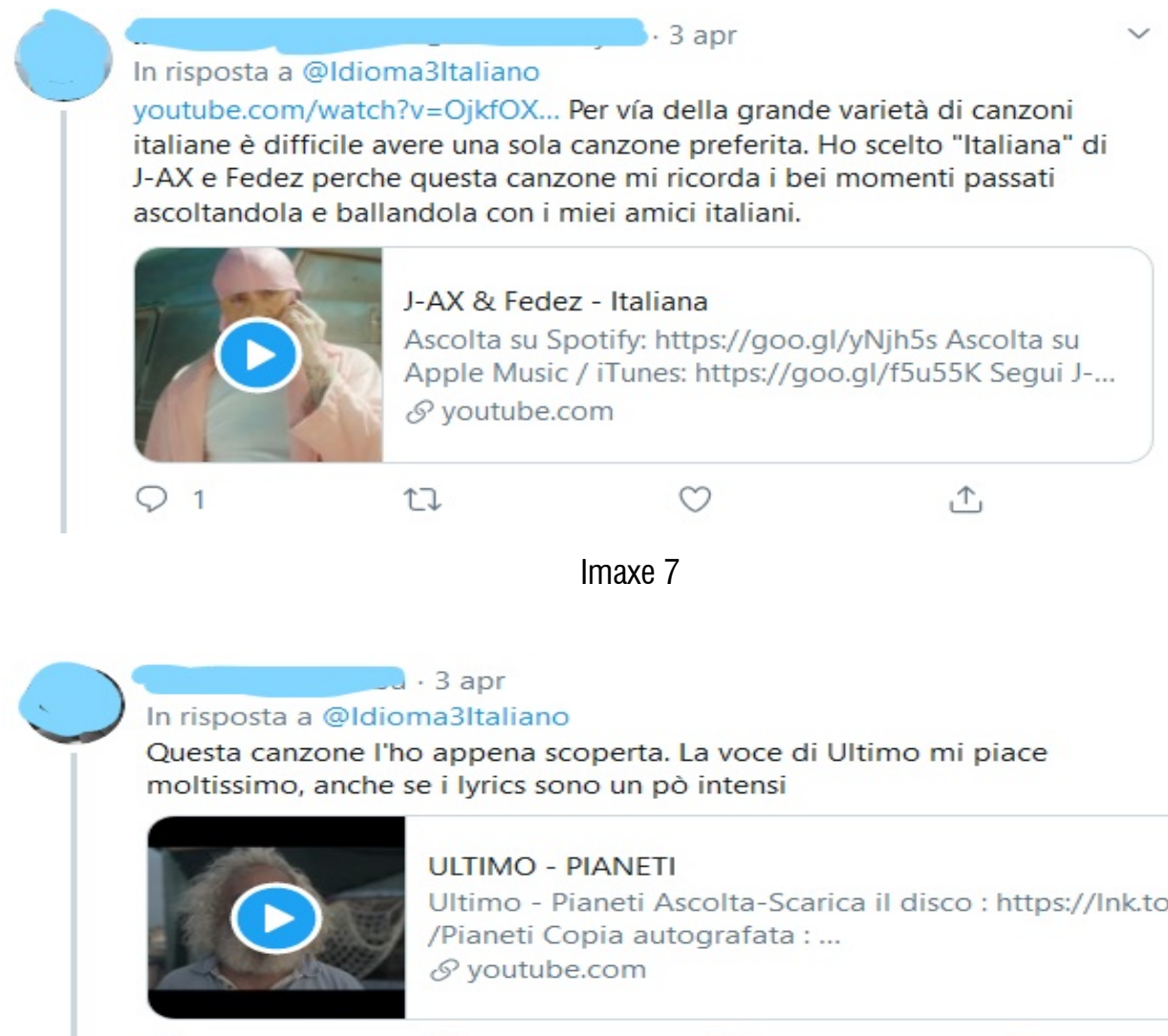

\section{3 apr}

In rispostaa@Idioma3Italiano

Questa canzone I'ho appena scoperta. La voce di Ultimo mi piace tissimo, anche se i lyrics sono un pò intensi

\section{ULTIMO - PIANETI}

Ultimo - Pianeti Ascolta-Scarica il disco : https://Ink.to /Pianeti Copia autografata : ...

(P) youtube.com

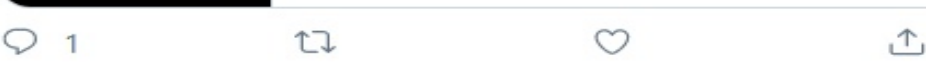

IdiomaModerno3:Italiano-18/19@Idioma3Italiano.3 apr I lyrics??? $\stackrel{\circ}{*}$
Q
七】
O
$\uparrow$
Il

\section{4 apr}

In risposta a@Idioma3Italiano

Non conosco molte canzoni italiane, ma questa mi piace molto perché mi ricorda quando ero piccola e mio padre adorava Eros Ramazzotti.

Anche ricordo che non sapevo come si diceva il suo nome e io lo chiamavo Eros Ramazmoti (i miei genitori ridevano molto)

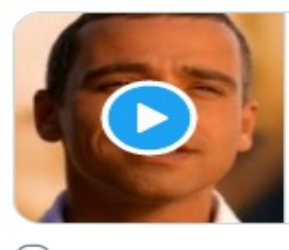

Eros Ramazzotti - Più Bella Cosa (videoclip)

Music video by Eros Ramazzotti performing Più Bella

Cosa. (C) 1997 BMG Ricordi Spa

(P) youtube.com

ऽ

七】

O

$\uparrow$

Imaxe 8 
Actividade 4: exercicio de creatividade

Seguindo co tema que se desenvolveu no inicio, a fraseoloxía, pensamos nunha actividade que mesturase 0 coñecemento das características dun proverbio e a creación. Desta maneira, os estudantes terán de inventar un proverbio e na imaxe 7 mostramos os resultados.

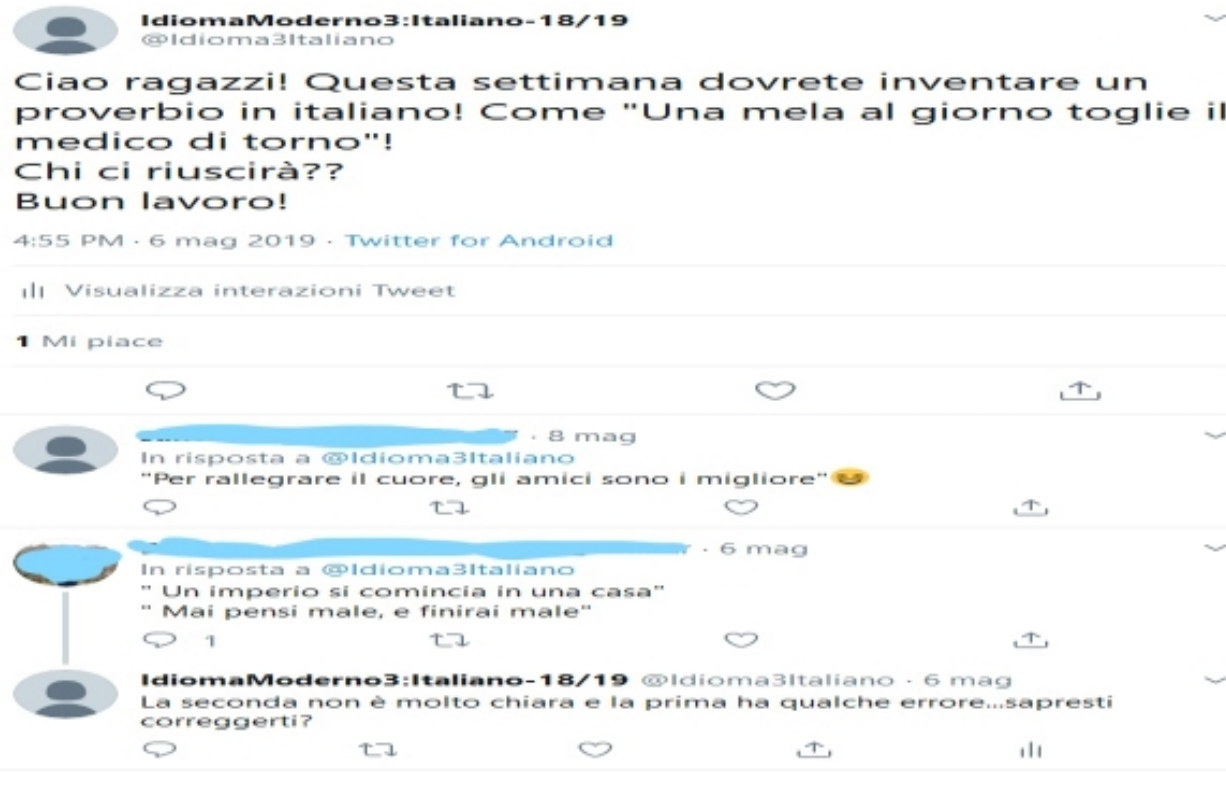

Imaxe 9

Actividade 5.0 ortonese a través da música

Un dos retos que o profesorado da lingua italiana debe ter en conta nas súas aulas é a introdución do tema da situación lingüística en Italia. As linguas rexionais teñen unha gran relevancia no ámbito da sociolingüística e representan unha realidade que non sempre se dá a coñecer ao alumnado. Por esta razón, fixemos un experimento co ortonese, falado na rexión de Abruzzo e pertencente ás linguas de Italia meridional. Para levar a cabo este exercicio, usamos unha canción da tradición popular ortonese e famosa en todo o país como himno desta rexión: "Vola vola", canción en ortonese composta por Guido Albanese e Luigi Dommarco no 1922. 
IdiomaModerno3:Italiano-18/19

@Idioma3Italiano

Eeeeeeh!!! Vola vola volaaaa! Ragazzi, sapreste dirmi qualcosa su questa canzone abruzzese? Avete capito di cosa parla? Commentate qualunque tipo di riflessione! Buon lavoro!

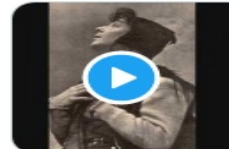

Vola Vola - Inno dell'Abruzzo

La bellissima canzone abruzzese composta dall'ortonese Luigi

Dommarco negli anni '20 del Novecento. Le illustrazioni sono...

\& youtube.com

1:12 PM - 28 apr 2019. Twitter for Android

Il Visualizza interazioni Tweet

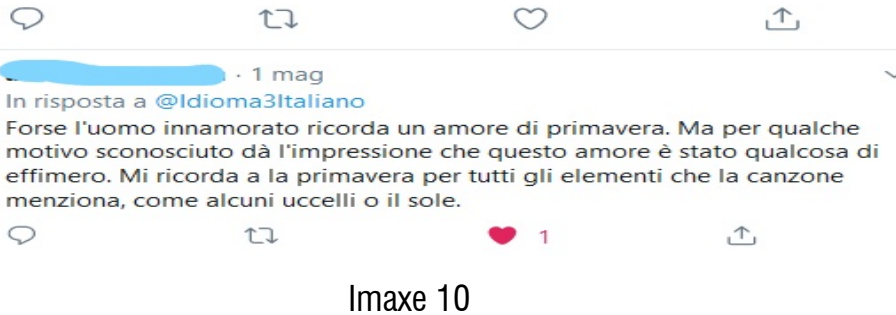

In risposta a @Idioma3Italiano

Per me, parla di un amore non corrisposto. Un huomo parla con la donna che ama ma lei non è interessata.
๑ 1
七】
1
$\uparrow$

IdiomaModerno3:Italiano-18/19@Idioma3Italiano.2 mag

Un huomo o un uomo??
$\odot$
$\urcorner$
O
$\uparrow$
Il

In risposta a@Idioma3ltalian

Penso di aver capito che il soggetto poetico é un ragazzo che parla dei ricordi con la donna che ama, ma il suo amore non è corrisposto. Mi sembra una poesia molto delicata 0
$\odot$
〔】
1
$\uparrow$

Imaxe 11

Para que a actividade for completa, retomamos a canción popular na aula e fixemos unha análise gramatical, léxica e textual da letra da canción. 


\section{RESULTADOS, CONCLUSIÓNS E UNHA OLLADA CARA AO FUTURO}

Os resultados acadados apuntan a que a experiencia do uso de Twitter na aula de italiano foi un medio de aprendizaxe que xunta, fundamentalmente, 0 aspecto lúdico e 0 didáctico. Os coñecementos que adquiriron os estudantes foi posíbel grazas a unha ferramenta que é parte das súas vidas e que empregan como medio de información e de diversión. Con Twitter é posíbel practicar contidos gramaticais, léxicos e de cultura á vez. Por medio da participación activa e constante dos alumnos a toda as propostas de traballo suxeridas pola docente, puidemos lograr os obxectivos formulados ao principio, insistindo constantemente sobre 0 uso didáctico desta ferramenta. A pesar de 0 non poder demostrar aínda, pensamos que 0 aspecto psicolóxico ten un papel considerábel neste tipo de actividades, xa que se comparamos os exercicios canónicos de gramática coas tarefas desenvolvidas no Twitter, podemos apreciar unha notábel mellora gramatical nas exposicións escritas. Dado que os caracteres dispoñibeis nesta rede social son reducidos, o alumnado vese obrigado a concentrar a información e a corrección do contido de xeito máis coidadoso. No entanto, remarcamos a idea de que estas metodoloxías de ensino non teñen nin deben substituír as aulas canónicas, pois os exercicios están pensados para reforzar o coñecemento, iso si, xuntando os aspectos gramaticais cos culturais.

Para o futuro queremos experimentar máis redes sociais, como, por exemplo, Instagram ou TikTok e comparar os resultados obtidos coas demais redes sociais e facer un estudo comparativo de cal delas é a máis acertada para o ensino do italiano como lingua extranxeira.

\section{REFERENCIAS}

Cerra, D. C. (2019). Las redes sociales y el aprendizaje de la lengua extranjera. Revista Boletín Redipe, 8(11), pp. 117-123.

Cotroneo, E. (2013). E-learning 2.0 per apprendere e insegnare l'italiano L2: i Social Network, Facebook e le tecniche didattiche. Italica Wratislaviensia, (4), pp. 37-57. 
Furini, M., \& Montangero, M. (2018). Sentiment analysis and Twitter: a game proposal. Personal and Ubiquitous Computing, 22(4), pp. 771-785.

Gobbis, A., \& Legler, M. P. (2011). Come l'arte può motivare l'interesse per apprendenti di lingua italiana L2. Italiano LinguaDue, 3(2), pp. 332-332.

Jacob, P., \& Uitdenbogerd, A. L. (2019). Readability of Twitter Tweets for Second Language Learners. Proceedings of the The 17th Annual Workshop of the Australasian Language Technology Association (pp. 19-27). Sydney, Australia: Australasian Language Technology Association

Kim, Y., Hwang, E., \& Rho, S. (2018). Twitter news-in-education platform for social, collaborative, and flipped learning. The Journal of Supercomputing, 74(8), pp. 3564-3582.

Mauroni, E. (2011). Imparare l'italiano L2 con le canzoni. Un contributo didattico. Italiano LinguaDue, 3(1), pp. 397-438.

Minghi, U. (2015). "Dal labbro il canto estasïato vola": Riflessioni linguistiche e glottodidattiche sull'italiano dell'opera lirica. Italiano LinguaDue, 7(1), pp. 131-156.

Ricoy, M. C., \& Feliz, T. (2016). Twitter as a learning community in higher education. Journal of Educational Technology \& Society, 19(1), pp. 237-248.

Rosell-Aguilar, F., Rosell-Aguilar, F., Beaven, T., \& Fuertes-Guiterrez, M. (2018). Twitter as a formal and informal language learning tool: from potential to evidence. Innovative Language Teaching and Learning at University: Integrating Informal Language into Formal Language Education, pp. 99-106.

Tang, Y., \& Hew, K. F. (2017). Using Twitter for education: Beneficial or simply a waste of time?. Computers \& Education, 106, pp. 97-118.

Valdiviezo, J. L. C., Viteri, J. C. R., \& Carrera, G. M. D. (2017). Twitter en la Educación. Revista Publicando, 3(9), pp. 238-249.

\footnotetext{
i Para o uso de Twitter na educación, véxanse, entre outros, Ricoy \& Feliz (2016), Tang, \& Hew (2017), Valdiviezo, Viteri \& Carrera (2017), Kim \& Rho (2018).

ii Algúns estudos sobre 0 uso de Twitter na aula de linguas son Cotroneo (2013), Furini \& Montangero (2018), Rosell-Aguilar, Beaven \& Fuertes-Guiterrez (2018), Jacob \& Uitdenbogerd (2019), Cerra (2019), entre outros.

iii Mauroni (2011), Gobbis \& Legler (2011), Minghi (2015), entre outros.
} 